Check for updates

Cite this: RSC Adv., 2018, 8, 21712

\title{
Bioprinting of 3D tissues/organs combined with microfluidics
}

\author{
Jingyun $\mathrm{Ma}^{\mathrm{ab}}$ Yachen Wang ${ }^{\mathrm{ab}}$ and Jing Liu (D) *ab
}

Accompanied by the increasing demand for organ transplants and personalized medicine, recent years have witnessed great developments in the regeneration of tissues/organs, which has benefited from various manufacturing technologies, especially 3D bioprinting. In 3D bioprinting, according to the morphogenesis, cellular microenvironment, and biological functions of the native tissues/organs, cells and biomaterials are printed by layer-by-layer assembly to form 3D bio-functional units. However, there are still substantial differences between existing 3D printed constructs and actual tissues and organs, especially in microscale structures such as vascular networks. By manipulating controllable fluids carrying biomolecules, cells, organisms, or chemical agents, microfluidic techniques aim to integrate biological or chemical functional units into a chip. With its features of biocompatibility, flexible manipulation, and scale integration on the micro/nanoscale, microfluidics has been a tool that has enabled the generation of micro-tissues/organs with precise configurations. With the inspiration of these two technologies, there have been efforts to fabricate functional living tissues and artificial organs with complex structures via a combination of 3D bioprinting and microfluidics, which may lead to unexpected effects. In this review, we discuss advances in microfluidics-assisted bioprinting in the engineering of tissues/organs and provide future perspectives for this combination in the generation of highly biomimetic tissues and organs in vitro.

\begin{abstract}
Received 9th April 2018 Accepted 5th June 2018 DOI: $10.1039 / \mathrm{c} 8 \mathrm{ra03022g}$ rsc.li/rsc-advances
\end{abstract}

\section{Introduction}

Currently, there is increasing demand for organ transplants as a treatment for various types of accident or disease. However, an

${ }^{a}$ Regenerative Medicine Center, The First Affiliated Hospital of Dalian Medical University, Dalian 116011, China. E-mail: liujing@dmu.edu.cn; Tel: +86-411-83635963-2170

${ }^{b}$ Stem Cell Clinical Research Center, The First Affiliated Hospital of Dalian Medical University, Dalian 116011, China imbalance remains between the supply of organs and the demand for organs, as reported by the Scientific Registry of Transplant Recipients in $2014 .^{1}$ Tissue engineering has emerged as a powerful tool for the recovery and regeneration of tissues and organs as candidates for organ transplantation with decreased side effects and immune responses, which are common problems with artificial mechanical organs and xenotransplants. ${ }^{2,3}$ Moreover, engineered tissues and organs could be used as in vitro physiological or pathological models for drug testing and screening. ${ }^{4}$ In particular, tissues and organs derived

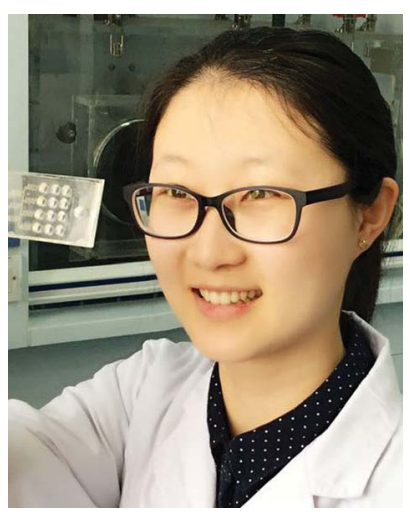

Jingyun $\mathrm{Ma}$ is presently an associate professor at the SinoUK Regenerative Medicine Center, the First Affiliated Hospital of Dalian Medical University, China. She achieved her PhD degree at Dalian Institute of Chemical Physics, Chinese Academy of Sciences, in 2014. Her research area of interest includes microfluidic related new technologies and application, functional biomaterial synthesis, and $3 D$ cell culture system construction.

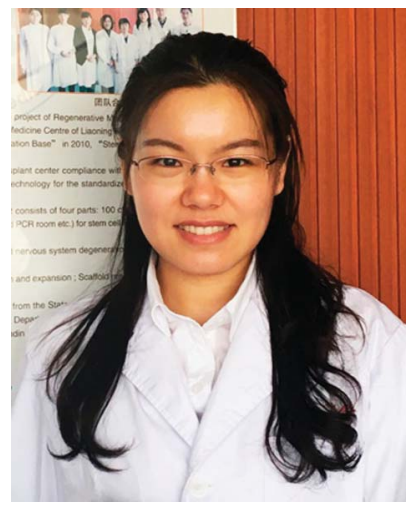

Yachen Wang is presently a doctor at the Sino-UK Regenerative Medicine Center, the First Affiliated Hospital of Dalian Medical University, China. She achieved her PhD degree from China Medical University in 2017. Her research area of interest includes neural stem cell transplantation, neural stem cell fate determination and microfluidic applications in stem cells. 
from the host are promising for personalized medicine, including customized tissue/organ implantation, predictive drug screening, and other effective regenerative therapies. ${ }^{5}$

3D bioprinting, which is a new type of tissue engineering technology, has come into the spotlight owing to its ability to produce biomimetic architectures. ${ }^{6,7}$ The aim of this technique is to fabricate 3D organized heterogeneous structures that are physiologically and morphologically similar to the relevant in vivo biological architectures. In this technology, cell-laden biomaterials are used as bioinks and raw materials. In the course of rapid prototyping and additive manufacturing, cells are deposited with directional control according to the respective design data. Via layer-by-layer accumulation, 3D tissues and organs are generated that are similar to the corresponding biological structures. Since the first bioprinting concept was proposed by Mironov et al. in $2003,{ }^{8}$ increasing numbers of research studies have attempted to develop biomimetic architectures using this technology. In comparison with conventional scaffold-based tissue engineering strategies, the advantages of 3D bioprinting lie in the following aspects., ${ }^{\mathbf{9 1 0}}$ Tissues/organs constructed by 3D bioprinting have higher resolution owing to the precise control of the spatial distribution of biomaterials and cells during the printing process, which helps to improve the biological activity and function of the tissues. These technologies enable one-step and smart combination of biomaterials, biological factors, and cells to integrate into one entity. Personalized printing of tissues/organs based on patients' physiological data can meet the specific demands of patients. Rapid printing processes enable the assembly of tissues/ organs in a very short period of time to ensure cell survival. With the development of 3D printing and biological technologies, 3D bioprinting technology has extended its field of application to the regeneration of complex tissues such as bone, cartilage, and blood vessels, as well as the $3 \mathrm{D}$ microstructure of complex organs including the liver, skin, etc. ${ }^{\mathbf{1 1 - 1 5}}$ However, reported printed tissue structures are unlike the presents in actual tissues, and most biological printing methods are still at the stage of shape control, rather than function control. Current bioprinting approaches still have many obstacles to overcome with respect to further

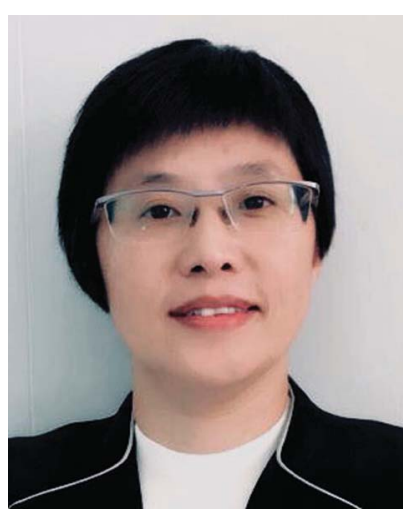

Jing Liu is currently a Professor in the First Affiliated Hospital of Dalian Medical University, China. She has been appointed as the Director of China's first batch of Stem Cell Clinical Research Institution, State-level Stem Cell Transplantation and Regenerative Medicine International Cooperation Base, and National \& Local Joint Engineering Laboratory. She has published more than 40 peer-reviewed papers and two books. Her current research interests are stem cell therapeutic strategies for nerve injury repair, and bionic synthesis of biological materials by microfluidic and $3 D$ bioprinting technologies. improvements in resolution, the construction of microscale structures for vascularization and innervation within complex 3D tissues, and the subsequent integration of culture and monitoring systems for printed tissues/organs.

In microfluidic techniques, namely, lab-on-a-chip approaches, controllable fluids carrying biomolecules/cells/organisms or chemical agents are manipulated with the aim of forming biological or chemical functional units within a chip. ${ }^{16}$ Owing to its essential features of biocompatibility, flexible manipulation, and scale integration on the micro/nanoscale, microfluidics has been regarded as a tool for enabling the generation of tissues/organs with precise configurations. ${ }^{17,18}$ In particular, organ-on-a-chip techniques have recently arisen as a new direction for development, in which human cells are confined in microchannels to form constructs, microtissues, or organoids with bio-functions. ${ }^{19,20}$ The properties of organ-on-a-chip confer great benefits in the simulation of human bodily responses, and they may become an alternative drug screening model, replacing some animal studies, in the near future. However, the most obvious disadvantage of microfluidic techniques in the regeneration of tissues/organs is the difficulty of scaling up microtissues or organoids, which leads to great differences in size from that of the actual tissues/organs.

Recently, combinations of bioprinting and microfluidics have been used to engineer 3D tissues/organs. These approaches could complement each other to create functional artificial tissues and organs with complex architectures via layer-by-layer assembly for the "growing up" of biological units by 3D bioprinting and regulation of precise structures on the micro/ nanoscale by microfluidics. In particular, 3D bioprinting may require the assistance of microfluidics for the construction of some microscale structures. To date, there have been several detailed reviews of $3 \mathrm{D}$ printed microfluidic devices, ${ }^{21-23}$ which mainly deal with the fabrication of microfluidic devices using 3D printing. However, no review has addressed the bioprinting of 3D tissues/organs in combination with microfluidics, and this is the first attempt to summarize this topic. Here, we initially introduce the basic operations used in 3D bioprinting and microfluidics for the generation of tissues/organs. Then, we divide bioprinting/ microfluidics combinations used for the construction of tissues/organs into three classes. According to the method employed to combine the two techniques, recent advances in the bioprinting of 3D tissues/organs in combination with microfluidics could be categorized into microfluidic modified printing nozzles, cell printing in the microfluidic receiving plate, and bioprinting of constructs with built-in microchannels. Finally, we provide future perspectives for this combination.

\section{Basic strategies involved in 3D bioprinting and microfluidics for generation of tissues/organs}

The main steps involved in 3D bioprinting include data acquisition (e.g., via computed tomography or magnetic resonance imaging), computer-aided 3D modeling (CAD-CAM), bioink preparation, and tissue structure printing. ${ }^{6,7}$ A thorough 
understanding of the structure and microenvironment of actual tissues/organs is the foundation of the replication of heterogeneous tissues and organs. Factors that should be taken into consideration include the distributions of functional and supporting cells, the concentrations of soluble and insoluble factors, the composition and mechanical properties of the extracellular matrix (ECM). The essential function of the bioink comprises loading cells that provide an outer supporting environment for cells loaded during the printing and culture processes. ${ }^{24}$ Matrix materials include synthetic and natural polymers and the ECM secreted by cells. The cell source should contain basic functional cells and cells that play roles in supporting the function and structure of the microenvironment. Current 3D bioprinting techniques can be divided into four categories on the basis of their working principles, namely, inkjet bioprinting, extrusion-based bioprinting, laser directwrite bioprinting, and stereolithographic bioprinting, as shown in Fig. 1a-d. Several detailed studies have covered the characteristics of these printing methods. ${ }^{25-27}$ Here, we briefly introduce their working principles. In inkjet bioprinting, also called "drop-on-demand" printing, bioink droplets are sprayed through an inkjet nozzle to predefined locations to produce a 2D cell pattern by layer-by-layer assembly and eventually form a $3 \mathrm{D}$ construct. The production of droplets can be triggered by pressure pulses induced by thermal or acoustic (piezoelectric) forces. Extrusion-based bioprinting utilizes pneumatic or mechanical (piston- or screw-driven) extrusion systems to extrude continuous fibres of cell-laden hydrogel bioinks. In laser direct-write bioprinting, a high-intensity laser is focused on an absorbent substrate to propel bioink droplets, which are dripped in noncontact mode. Using the principle of photopolymerization, stereolithographic bioprinting employs a scanning laser beam to selectively transform liquid photosensitive bioinks into the solid state in a layer-by-layer manner.

The use of microfluidic technology to design and prepare functional $3 \mathrm{D}$ cell-laden constructs has recently become a popular topic. According to the definition of 3D materials in our published review, ${ }^{28}$ which was specific to $3 \mathrm{D}$ cell-laden constructs produced via microfluidics, this term refers to materials with sizes in each dimension of almost the same order of magnitude that exhibit a "construct" appearance, and the sizes in at least two dimensions are on the millimeter scale. Microfluidic syntheses of 3D cell-laden constructs usually adopt a hydrogel assembly strategy, including the formation of hydrogel constructs with tunable geometries via microchannel constrictions, ${ }^{29}$ bottom-up engineering of hydrogel building blocks, ${ }^{30}$ and the assembly of 3D constructs from microfluidic spun microfibres (e.g., via reeling, weaving, or direct writing), ${ }^{31-33}$ as shown in Fig. 1e-g. However, research works on microfluidic $3 \mathrm{D}$ cell-laden constructs are relatively scarce owing to the increased complexity of the microfluidics design and operation needed for the preparation of $3 \mathrm{D}$ materials.

\section{Microfluidic modified printing nozzles}

As a novel biofabrication method, 3D bioprinting still suffers from some limitations, which relate to: (i) the ability to deposit multiple biomaterials/cells into a single construct; (ii) the ability to print precise configurations; and (iii) the possibility of changes in cell phenotype due to damage from heat or mechanical force during the printing process. In these cases, microfluidic modified printing nozzles with specific designs could facilitate the deposition of the bioink to create intra-complex architectures within a single bulk. It is possible to enhance the accuracy and printing quality to a degree that was previously unachievable. In addition, in comparison with commonly used bioprinting heads, a microfluidic printing nozzle can offer a mild fabrication environment, which is assumed to protect cells from damage induced by the printing process. In particular, microfluidic printing heads are compatible with micro/nanometer-scale synthesis, and the resulting printed structures may match blood vessels, nerve and muscle units.

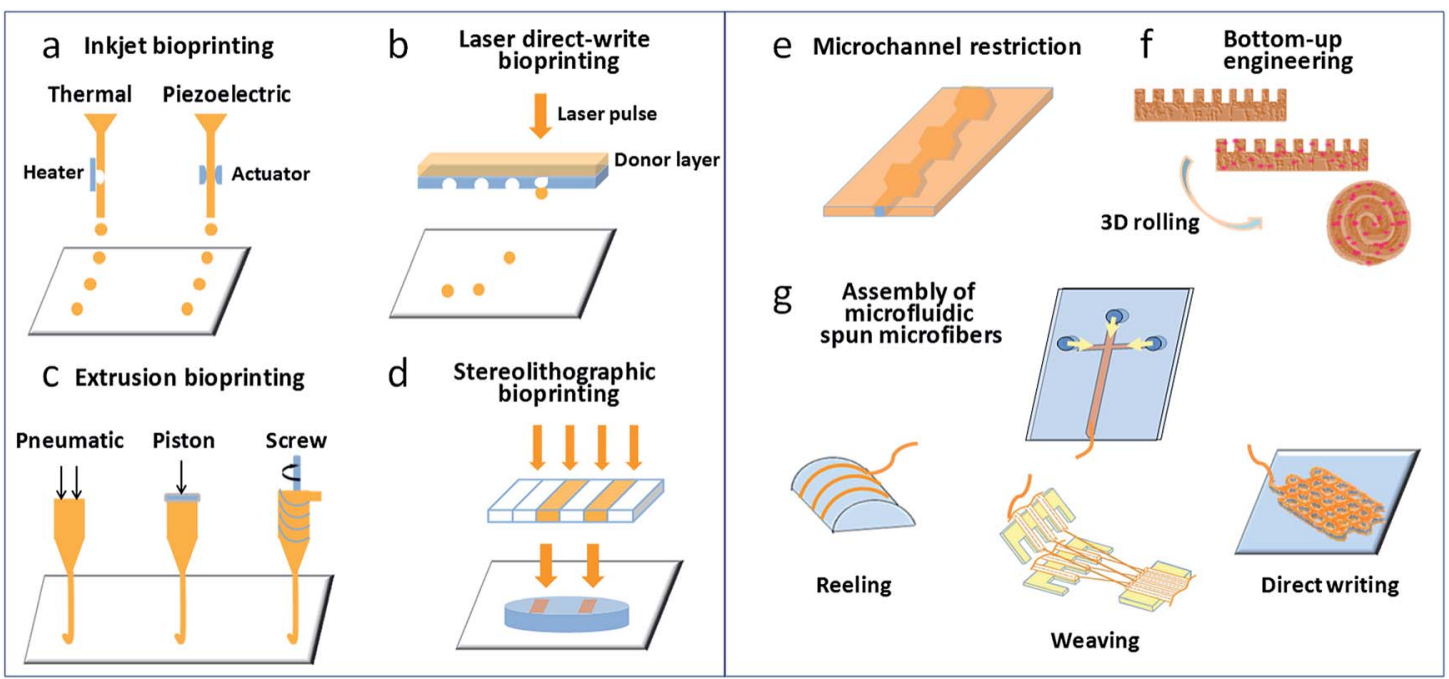

Fig. 1 (a-d) Bioprinting techniques most commonly used for the generation of tissues/organs; (e-g) microfluidic approaches involved in creating tissues/organs. 
Vascularization provides functions to supply nutrients and oxygen to, and remove waste from, living tissues and organs. The resulting network system should feature sufficient mechanical strength and elasticity, and high perfusability. ${ }^{34}$ However, vascularization in engineered tissue/organ constructs remains a major challenge. With the assistance of microfluidic printing heads, vascularized tissue/organ constructs that consisted of hollow fibres mimicking blood vessels and cell constructs encapsulated in the fibre walls could be successfully bioprinted. Zhang et al. utilized a pressure-assisted coaxial microfluidic needle to print vessel-like hollow hydrogel filaments with the potential to be used in the fabrication of vascularized tissues/organs. These channels could not only support mechanical integrity but also enable the transport of fluid to a cellular assembly in a 3D cellular environment. Cartilage progenitor cells encapsulated in the hydrogel wall could maintain high viability during prolonged culture in vitro and exhibit cartilage-producing functions..$^{35}$ Similarly, with the aim of constructing vascularized organs, Dolati et al. printed vascular conduits reinforced with carbon nanotubes (CNTs), in which the CNTs enhanced the mechanical properties, perfusability, permeability, bioprintability, and biocompatibility of the materials, as confirmed by the culture of human coronary artery smooth muscle cells. More importantly, these synthesis conduits may guide biomaterials reinforced with natural protein nanofibres for the integrated fabrication of large-scale tissue constructs. ${ }^{36}$ Gao et al. developed high-strength cellladen hydrogel structures with internal microchannels by 3D bioprinting with the assistance of a coaxial microfluidic nozzle, as shown in Fig. 2a. ${ }^{37}$ The adjacent hollow calcium alginate filaments that were extruded could be fused by controlling the crosslinking time and incorporated into a hydrogel block. Fig. 2a(i) shows that sodium alginate solution and calcium chloride solution were distributed through the outer and inner of the coaxial nozzle, respectively. Perfusion test in the printed hollow filament in Fig. 2a(ii) displays the feasibility of nutrients media supply. Under the fusion effect of adjacent alginate hollow filaments, the printed structure consisting of multilayers of hollow filaments seems to be a unified whole with built-in microchannels (Fig. 2a(iii)). The viability of encapsulated fibroblasts in the filaments confirms the biocompatibility of this method (Fig. 2a(iv)). Gao et al. also generated 3D hydrogel-based vessel-like structures with macrofluidic channels by rotating hollow alginate filaments with microfluidic channels, as shown in Fig. $2 \mathrm{~b}^{38}$ The microchannels were loaded with smooth muscle cells and fibroblasts (Fig. 2b(i)) and extruded through a coaxial nozzle. Meanwhile, endothelial cells were seeded into the inner wall of the macrofluidic channels (Fig. 2b(ii)). Fig. 2b(iii) shows the structure of macrofluidic channels with outer microfluidic channels. Using this system, vascular circulatory flow, simulations of cerebral artery surgery, and cell co-culture could be achieved. Fig. 2b(iv) displays coculture of L929 mouse fibroblasts, smooth muscle cells, and endothelial cells in this printed vessel-like structure. Attalla et al. developed an instantly perfusable vascular network combined with cell-laden gel scaffolds, as depicted in Fig. 2c. ${ }^{39}$ A hollow structure of artificial vascular tubes was generated using a microfluidic printing head with coaxial geometry via the extensive diffusion of interior calcium ions into a surrounding annular alginate phase (Fig. 2c(i)). These tubes could be assembled into scaffolds or tissue constructs using a 3D printing system. The hollow channels could also be embedded within calcium alginate constructs loaded with Escherichia coli (Fig. 2c(ii)) or human umbilical vein endothelial cells (HUVECs, Fig. $2 \mathrm{c}(\mathrm{iii})$ ). The perfusion of medium for HUVECs facilitated by the channels caused a significant increase in cell viability in comparison with that in non-vascularized bulk gels. This approach enabled a wide range of cells, growth factors, and ECM materials to combine to create a potential alternative vascular network.

Besides the fabrication of vascularized organs, microfluidic modified printing has also been employed in other contexts. Ghorbanian et al. used a microfluidic direct writer to fabricate cell-laden hydrogel constructs with openings that permitted exchange of medium, as shown in Fig. 2 d. $^{40}$ By computer-aided layer-by-layer bioprinting through a microfluidic printing head in coaxial flow format (Fig. 2d(i)), 3D multilayer constructs assembled from calcium alginate fibres (Fig. 2d(ii)) or from fibres loaded with HEK-293 cells could be generated (Fig. 2d(iii)). Costantini et al. used customized microfluidic enhanced bioprinting for the alignment of myoblast-laden hydrogel bulks as organized myofibers and then as artificial skeletal muscle tissue, ${ }^{41}$ as shown in Fig. 2e. The microfluidic bioprinting head featured a multi-inlet Y-junction and a coaxial geometry (Fig. 2e(i)). Fig. 2e(ii) shows the bioprinted construct composed of unidirectional aligned PEG-fibrinogen fibres. With the assistance of the microfluidic printing head and its variants, 3D multicellular assemblies compartmentalized into different types of encapsulated cells could be fabricated by extruding different bioinks simultaneously or by rapidly switching the bioink. The ability to engineer hydrogels containing muscle precursor cells to form long-range multinucleated myotubes in parallel alignment (Fig. 2e(iii)) and, subsequently, macroscopic artificial muscle for scaling up skeletal muscle tissue has potential applications in muscle repair.

\section{Cell printing in the microfluidic receiving plate}

Bioprinting is the process of printing cells directly and assembling them into an organized structure, which implies that the printed constructs should be followed by in vitro culture and detection of their biological function. At present, these subsequent culture and analysis processes are independent. Bioprinting technology will be better employed if it integrates these two processes. The concept of organ-on-a-chip refers to the creation, culture, and analysis of artificial living organs on a chip that mimic the physiological responses of real organs. ${ }^{42}$ With this as an inspiration, researchers have attempted to utilize microfluidic channels and chambers as the receiving plate, i.e., to carry out 3D printing on a chip. This method can complete the printing and culture of, administration of stimuli 

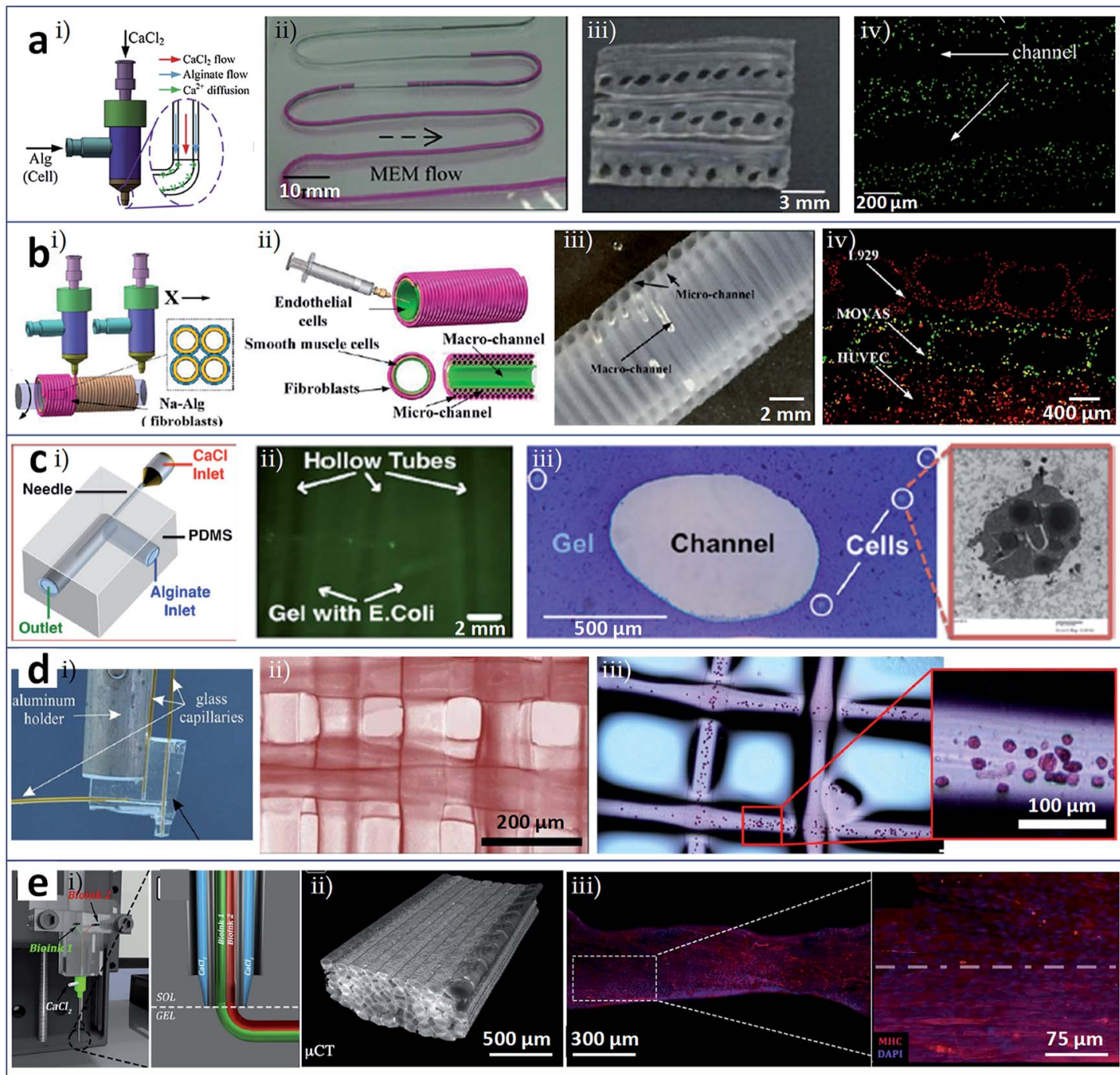

Fig. 2 3D bioprinting of tissues/organs assisted by microfluidic modified printing head. (a) Coaxial nozzle for printing cell-laden 3D hydrogel structures composed of vessel-like perfusable filaments. (i) Solution distributions in the coaxial nozzle; (ii) perfusion test of cell culture media in the printed hollow filament; (iii) the cross-section of the adjacent alginate hollow filaments; (iv) fused channel structure and encapsulated cells. (b) Coaxially printed constructions with multilevel fluidic channels. (i) Smooth muscle cells and fibroblasts loaded in the microchannels; (ii) endothelial cells seeded onto the inner wall of the macrofluidic channels; (iii) the structure of macrofluidic channels with outer microfluidic channels; (iv) vascular cells (L929 mouse fibroblasts, smooth muscle cells, and endothelial cells) co-cultured in this printed vessel-like structure. (c) Gels with channels formed by 3D printing with a microfluidic nozzle. (i) Coaxial geometry of the microfluidic printing head; (ii) Escherichia coli and (iii) HUVECs loaded in the constructs with hollow channels. (d) Microfluidic direct-write bioprinting for fabricating cell-laden hydrogel constructs. (i) Microfluidic printing head in a coaxial flow format; (ii) and (iii) 3D multilayer constructs assembled from calcium alginate fibres and fibres loaded with HEK-293 cells. (e) Microfluidic enhanced bioprinting for fabricating functionally organised myofibers. (i) Microfluidic bioprinting head featured a multi-inlet Y-junction and a coaxial geometry; (ii) bioprinted construct composed of aligned PEG-fibrinogen fibres; (iii) myotube in parallel alignment obtained from 3D bioprinted constructs after 15 days of in vitro culture. Reproduced with permission from ref. $37-41$.

to, and detection of the function of 3D constructs. Meanwhile, this process achieves the construction of an organ-on-a-chip.

Chang et al. fabricated a 3D liver-on-a-chip as an in vitro model of drug metabolism. The model involved the construction of a 3D liver architecture via a direct cell writing process, its integration onto a microfluidic device in defined design patterns for perfusion culture, and the assessment of liver cellspecific functions and drug metabolism capacity. ${ }^{\mathbf{4 3 , 4 4}}$ Matsusaki et al. achieved the layer-by-layer assembly of hepatocytes and endothelial cells at the single cell layer level using inkjet printing and a microfluidic receiving plate, ${ }^{45}$ as shown in
Fig. 3a. The number of layers and the cell types in the resulting micrometer-sized 3D liver cell arrays could be controlled. As indicated from the photograph and illustration in Fig. 3a(i), HepG2 monolayer (1L), HUVEC/HepG2 (2L), and HUVEC/ HepG2/HUVEC (3L) hepatic co-culture arrays could be fabricated in micro-well plates, and those tissues were labelled with albumin expression. This 3D liver tissue chip could be employed in high-throughput drug evaluation. Fig. 3a(ii) displays cytotoxicity of troglitazone to $1 \mathrm{~L}, 2 \mathrm{~L}, 3 \mathrm{~L}$ constructs and HUVEC monolayer. Bhise et al. developed a liver-on-a-chip system by integrating bioprinted hepatic spheroids onto 
a chip, ${ }^{46}$ as shown in Fig. 3b. Fig. 3b(i) displays the hepatic bioreactor integrated with a 3D bioprinter and a biomarker analysis module. Fig. 3b(ii) shows printing of dot arrays composed of hydrogel-based hepatic constructs in the bioreactor. This platform enabled not only the long-term perfusable culture of human HepG2/C3A spheroids with maintenance of their function (Fig. 3b(iii)) but also the in situ monitoring of cell behaviors. An assessment of acetaminophen toxicity demonstrated the use of this liver-on-a-chip as a model for predicting drug toxicity. Snyder et al. developed two types of cell-laden microfluidic devices, one as a mold for the direct printing of a cell-laden matrix into the open channels, and the other as a scaffold to support and guide the growth of HepG2 cells. ${ }^{47}$ Zhang et al. used inkjet bioprinting for microscale multiple cell patterning in the microfluidic receiving plate for the establishment of a model of drug metabolism and diffusion, as shown in Fig. 3c. ${ }^{48}$ Cell arrays were patterned on glass slides, which were covered by a layer of polydimethylsiloxane (PDMS) with corresponding connecting microchannels (Fig. 3c(i)). Copatterned hepatoma and glioma cells were used for the drug metabolism and diffusion tests. Fig. 3c(ii) indicates HepG2 cells metabolized tegafur exhibited an anticancer effect on U251 cells by evaluation of the viability of HepG2 and U251 co-culture system.

\section{Bioprinting of constructs with built-in microchannels}

Vascularization is not only essential to maintain tissue/organ activity but is also one of the most fundamental challenges in

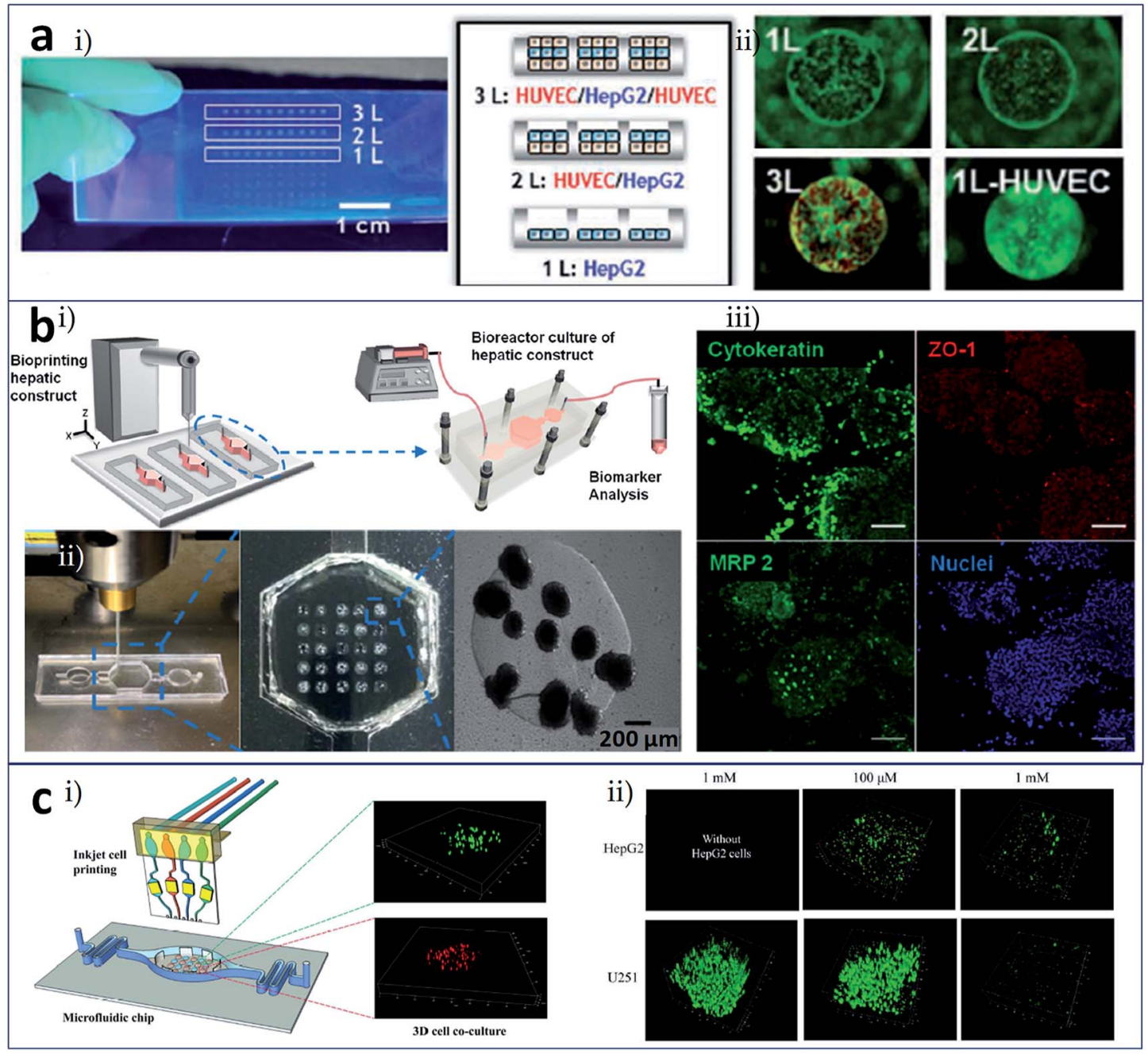

Fig. 3 Cell printing in the microfluidic receiving plate for the construction of tissues/organs. (a) 3D tissue chips formed by inkjet cell printing. (i) Photograph and illustration of the HepG2 monolayer (1L), HUVEC/HepG2 (2L), and HUVEC/HepG2/HUVEC (3L) hepatic co-culture system in micro-well plates; (ii) cytotoxicity of troglitazone on $1 \mathrm{~L}$ to $3 \mathrm{~L}$ constructs and HUVEC monolayer. (b) Liver-on-a-chip system facilitated by bioprinted hepatic spheroids. (i) The hepatic bioreactor integrated with a 3D bioprinter and a biomarker analysis module; (ii) printing of dot arrays composed of hydrogel-based hepatic constructs in the bioreactor; (iii) HepG2/C3A spheroids with maintenance of their function (cytokeratin, ZO-1 tight junction binding protein, and MRP-2 biliary canalicular transporter immunostained spheroids cultured for 30 days), scale bars are 100 $\mu \mathrm{m}$; (c) controlled printing of multiple cells in a microfluidic chip for drug metabolism tests. (i) Cell arrays patterned on glass slides integrated with a layer of the microfluidic plate; (ii) tegafur metabolism assay by evaluation of the viability of HepG2 and U251 co-culture system. Reproduced with permission from ref. 45,46 and 48 . 
tissue engineering. ${ }^{49}$ Various kinds of blood vessels extend over tissues and organs that have different sizes (diameters from the micrometer to the centimeter scale) and hardnesses and are composed of different types of cell. Even with advanced bioprinting techniques, vascularization remains an elusive goal, which has led to the failure of the effective transportation of regional nutrition, subsequent cultivation, and scale-up to form organs. Microfluidic assisted bioprinting can help to generate a multiscale hydrogel-based flow network, in which flow structures on the micrometer and centimeter scales could be formed simultaneously with forms and functions that are closer in similarity to those of real blood vessels. In addition, the bioprinting of cellular constructs together with complex 3D microfluidic networks could facilitate the fabrication of microfluidic chips with a biocompatible hydrogel as the substrate material and the loading of various cellular microenvironments within the chip.

Two main approaches are used for blood vessel engineering: direct printing of separate tubular structures and indirect printing of a flow network inside the scaffolds used as organ supports. In the usual state of blood vessels in vivo, they are embedded in tissues and organs. Therefore, the indirect printing of a flow network inside the scaffolds used as organ supports, i.e., printing cell-laden hydrogel bulks with built-in channels, can better simulate the actual vascular microenvironment. The printing methods that are most commonly used for the construction of such built-in flow networks include sacrificial layer-based bioprinting, stereolithographic printing, and block assembly methods.

In bioprinting based on a sacrificial layer process, different sacrificial materials are available. Wu et al. deposited 3D filament networks within a photocurable hydrogel reservoir in an omnidirectional manner avoiding layer-by-layer patterning. They chose Pluronic F127 as the fugitive ink, which is liquid at low temperatures and solid at high temperatures, and this feature was used to achieve the exposure of the network. Hierarchical branching networks could be generated in this system. ${ }^{50}$ Miller $e t$ al. selected a printable cytocompatible sugar as the sacrificial layer material to produce microchannels that incorporated a hydrogel structure (Fig. 4a). ${ }^{51}$ The entire process included utilizing an extrusion method to print the 3D sugar supporting network, embedding the sugar scaffold into a cellladen hydrogel material, and dissolving the sugar to expose a hollow channel network (Fig. 4a(i)). Fig. 4a(ii) shows HUVECs lined alone the vascular space and 10T1/2 cells distributed in the fibrin bulk. Lee et al. initially printed a layer of collagen and then a cell-gelatin fibre and another layer of collagen. Finally, the gelatin was heated to melt it, and channels were exposed (Fig. 4b(i)). They seeded endothelial cells in internal channels and investigated morphological differences in the endothelial cells under dynamic and static culture. The fabricated vasculature with a confluent endothelial lining represented a barrier for specific biological substances. Fig. $4 \mathrm{~b}$ (ii) shows characterizations of the printed vascular channel by lined endothelial cells and the laminar flow in the channel by the motion of green fluorescent beads. ${ }^{52}$ They also constructed fluidic vascular channels with a lumen size of up to $1 \mathrm{~mm}$ and a microvascular bed between two large fluidic vessels for the observation of angiogenic sprouting. ${ }^{53}$ Bertassoni et al. chose agarose as the sacrificial layer material to achieve the construction of a network in a photosensitive cell-laden hydrogel bulk by directwrite bioprinting (Fig. 4c). ${ }^{54,55}$ The preparation process included bioprinting of agarose template fibres, casting and crosslinking of the hydrogel bulk over the template mould, removal of the template, and exposure of the fully perfusable microchannels, as shown in Fig. 4c(i). Fig. 4c(ii) shows bioprinted agarose templates (green, 3D branching and 3D lattice) embedded in hydrogel bulks and the resulting network perfused with a fluorescent microbead (red, 3D branching and 3D lattice). Also using agarose as the sacrificial material, Massa et al. bioprinted vascularized liver tissue to study drug toxicity, whereby HepG2/ C3A cells were encapsulated in a hydrogel bulk and HUVECs were seeded into microchannels. ${ }^{56}$ Kolesky et al. studied a multi-nozzle extrusion bioprinting device, in which one nozzle was used to print the sacrificial layer material (Pluronic F127) and the other nozzle was used to print a gel material containing cells (Fig. 4d). ${ }^{57,58}$ For the osteogenic differentiation of thick vascularized tissue, human mesenchymal stem cells (hMSCs) pervaded surrounding the vascular architecture and human neonatal dermal fibroblasts (hNDFs)-ECM filled the interstitial space (Fig. $4 \mathrm{~d}(\mathrm{i})$ ). After 30 days of perfusion culture and in situ differentiation, hMSCs in the vascularized construct expressed osteocalcin (Fig. 4d(ii)).

Stereolithographic printing is an efficient manufacturing method that is often used for printing microstructures of flow networks owing to its high print accuracy. Zhu et al. utilized a digital optical projection method to print a complex 3D prevascularized network on the microscale, as shown in Fig. 5a(i). ${ }^{59}$ In this process, there was no need of supports and sacrificial layer materials, and different kinds of cells could be deposited at precisely the same time. Hepatocellular carcinoma cells and endothelial cells were encapsulated in a gel and printed into blocks with a structure comprising channels with a diameter gradient. Fig. 5a(ii) shows heterogeneous tissue constructs in this method with HUVECs along the channels and HepG2 in the surrounding area, and Fig. 5a(iii) shows a 3D imaging of the endothelial cells which are located along the microchannel walls. Ma et al. established a hepatic model derived from human induced pluripotent stem cells (hiPSCs) via stereolithographic bioprinting. A two-step bioprinting process was used to build a 3D microscale hexagonal lobular structure by patterning hiPSC-derived hepatic progenitor cells in a gelatinmethacrylate hydrogel, as well as a vascular structure by patterning supporting cells surrounding the lobular structure. This model exhibited great potential in patient-specific drug screening and disease studies. ${ }^{60}$

Tissues and organs are composed of numerous basic functional modules or microscale tissue modules, which can be regarded as the smallest structural and functional units in their organization. In theory, complete organizations, such as builtin flow networks, could be constructed via the rational design and self-assembly of these functional modules. Norotte $e t$ al. utilized the mutual fusion of multicellular modules to construct tubular structures ${ }^{61}$ as shown in Fig. 5b. Firstly, cells were 


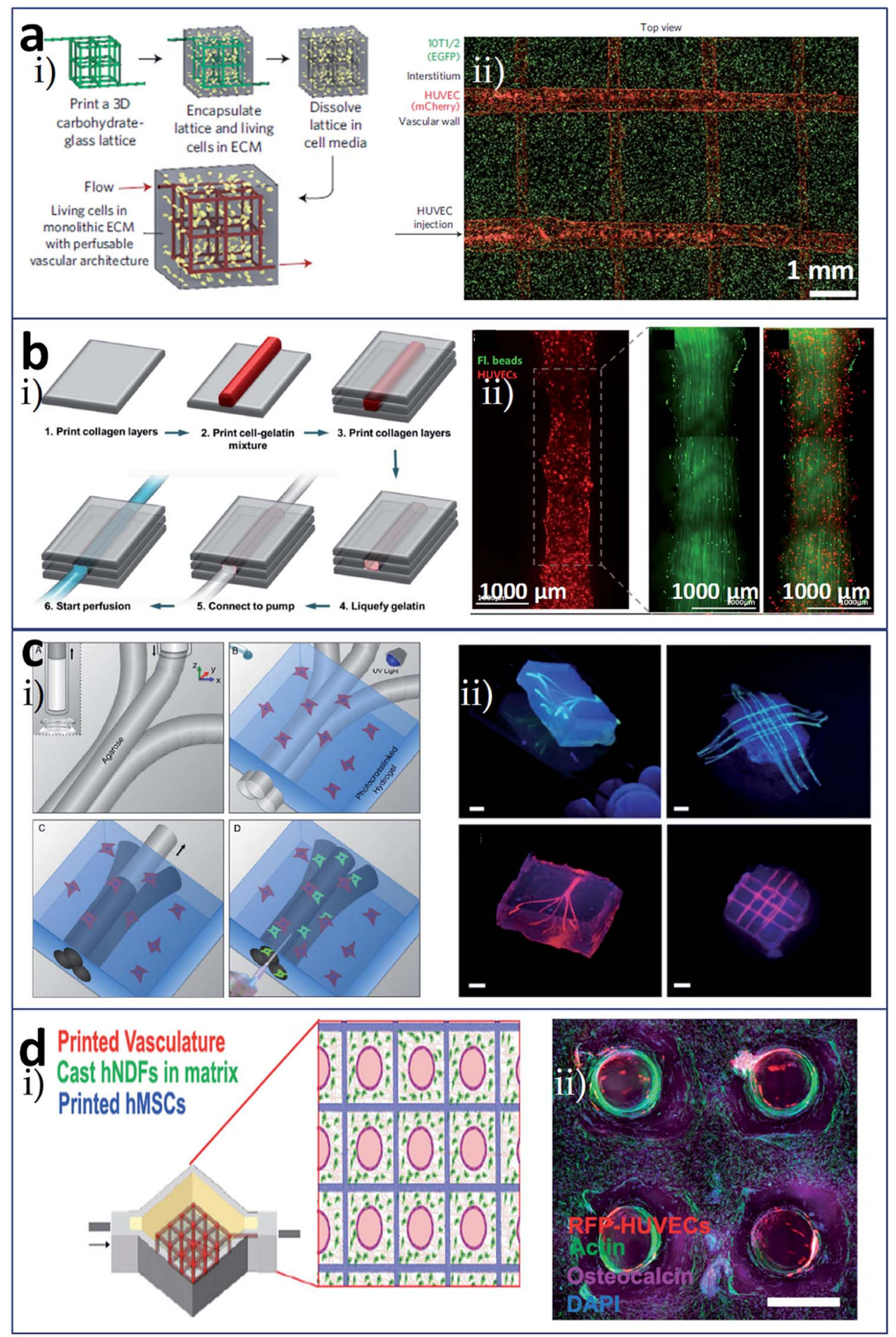

Fig. 4 Sacrificial layer-based bioprinting of tissues/organs with built-in microchannels mimicking blood vessels utilizing (a) sugar, (b) gelatin, (c) agarose, and (d) Pluronic F127, respectively, as the sacrificial layer material to produce vascular networks for perfusable tissues. (a-i) The entire preparation process included utilizing an extrusion method to print the 3D sugar supporting network, embedding the sugar scaffold into a cellladen hydrogel material, and dissolving the sugar to expose a hollow channel network; (a-ii) HUVECs lined alone the vascular space and 10T1/2 cells distributed in the fibrin bulk. (b-i) Vascular construction procedure from cell-gelatin mixture; (b-ii) characterizations of the printed vascular channel by lined endothelial cells and the laminar flow in the channel by the motion of green fluorescent beads. (c-i) Preparation process including bioprinting of agarose template fibres, casting and crosslinking of the hydrogel bulk over the template mold, removal of the template, and exposure of the fully perfusable microchannels; (c-ii) bioprinted agarose templates (green, 3D branching and 3D lattice) embedded in hydrogel bulks and the resulting network perfused with a fluorescent microbead (red, 3D branching and 3D lattice), scale bars are all $3 \mathrm{~mm}$. (d-i) Schematic diagram of heterogeneous tissue construction, in which hMSCs pervaded surrounding the vascular architecture and hNDFs-ECM filled the interstitial space; (d-ii) cross-section image of a vascularized osteogenic construct with a thickness of $1 \mathrm{~cm}$ after 30 days of perfusion culture and in situ differentiation, scale bar is $1.5 \mathrm{~mm}$. Reproduced with permission from ref. 51, 52, 55 and 57. 


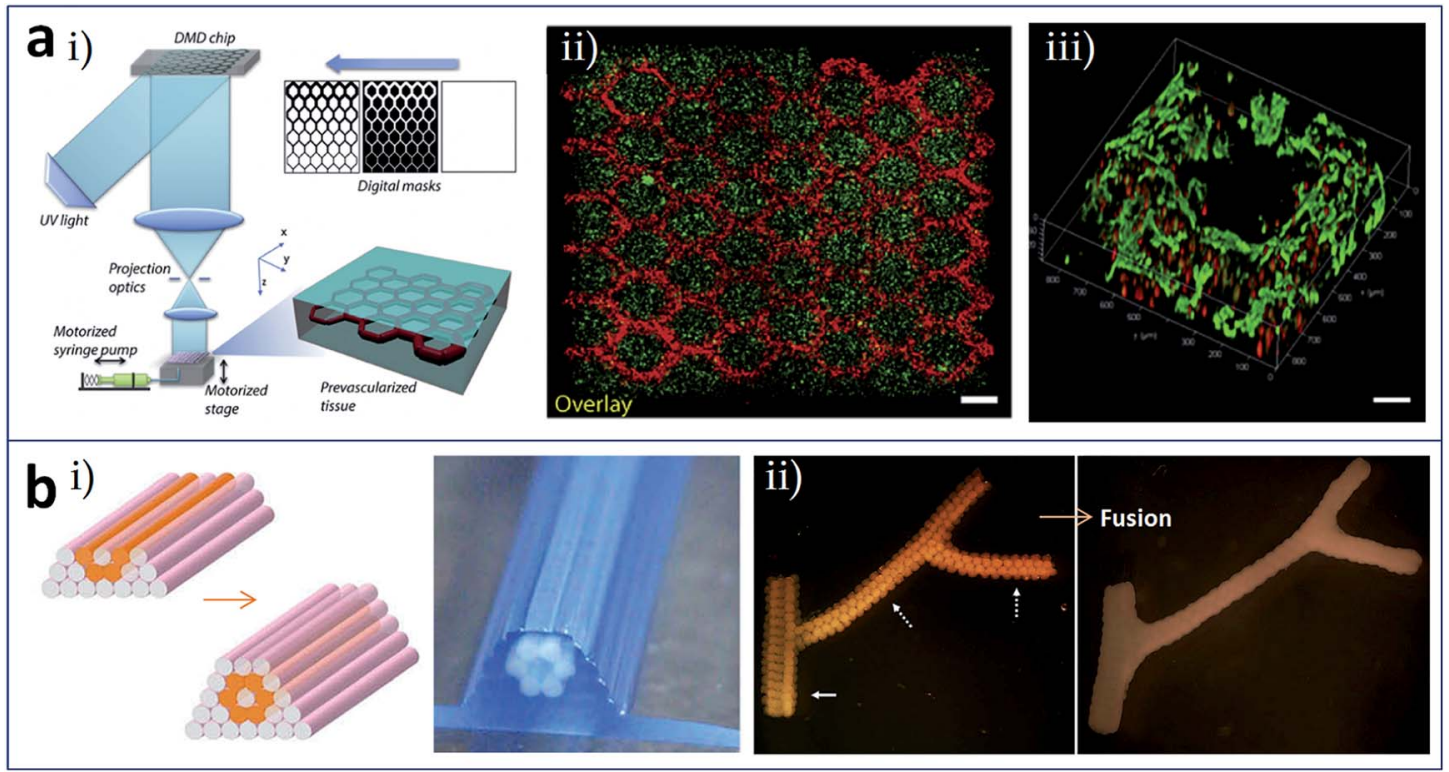

Fig. 5 (a) Stereolithographic printing and (b) construct assembly-based bioprinting of tissues/organs with built-in microchannels mimicking blood vessels. (a-i) Schematic of the stereolithographic printing of prevascularized tissue constructs; (a-ii) heterogeneous tissue constructs with HUVECs (red) along the channels and HepG2 (green) in the surrounding area, scale bar is $250 \mu \mathrm{m}$; (a-iii) 3D imaging of the endothelial cells which are located along the microchannel walls, red and green colors represent fluorescent cell tracker and CD31, respectively, scale bar is $100 \mu \mathrm{m}$. (bi) Design template and the printed construct of tubular structures with cellular cylinders; (b-ii) fusion of spheroids in the branched construct after 6 days of deposition. Reproduced with permission from ref. 59 and 61.

processed to form discrete units such as multicellular spheroids and multicellular cylinders. Then, these discrete modules were printed and assembled in a layer-by-layer manner using agarose rods as a supporting template. Vascular tubes (Fig. $5 \mathrm{~b}(\mathrm{i})$ ), even with complex branching structures (Fig. 5b(ii)), could be manufactured by this method.

Besides vascularization, analogous built-in network constructs such as renal tubules ${ }^{62}$ and nerve conduits ${ }^{63}$ have been reported. Using Pluronic F127 as the sacrificial material, Homan et al. printed 3D convoluted renal proximal tubules with an open lumen surrounded by the epithelial lining of the proximal tubule (Fig. 6a). Fig. 6a(i) shows schematic and photograph of the printing step during the preparation of tubules, in which Pluronic F127 is printed on a gelatinfibrinogen ECM. These proximal tubules were embedded in ECM and housed within a perfusable tissue chip. Features of proximal tubule models, including 3D convolutions, open lumen architectures, perfusion under physiological shear stresses, and longevity, were taken into consideration during the proof-of-concept demonstration. The 3D open lumen structure of the prepared tubule was verified in Fig. 6a(ii), which was circumscribed by an epithelial lining and could be directionally perfused on a chip. $\mathrm{Na} / \mathrm{K}$ ATPase, acetylated tubulin and nuclei were stained. Besides, molecular markers of primary cilia in apical side of the tubule, and actin + AQP1 in proximal tubule were highlighted in Fig. 6a(iii) and (iv). Owens et al. developed a layer-by-layer bioprinting method for the construction of a fully cellular nerve graft (Fig. 6b). The types and arrangement of cylinders used could be controlled. For nerve graft fabrication, bioinks composed of bone marrow stem cells (BMSC), cylinders comprised of $90 \%$ BMSC and 10\% Schwann cells
(SCs), and supporting agarose rods were alternately patterned as shown in Fig. $6 \mathrm{~b}(\mathrm{i})$, which produced multiple lumina inside of the graft. After fusion of the discrete bioink cylinders into a nerve graft, the supporting agarose rods were removed. The graft composed exclusively of cellular components was used for regeneration testing in a rat model of nerve injury. As shown in Fig. $6 \mathrm{~b}(\mathrm{ii})$, in the fabricated graft, more axons appeared close to the lumina, where the SCs were located.

\section{Summary and outlook}

Despite the advances made with 3D bioprinting in tissue engineering, some limitations remain, such as the inability to create tissue constructs containing elaborate microstructures for appropriate vascularization/innervation or to integrate the subsequent culture and analysis steps. Bioprinting of 3D tissues/organs in combination with microfluidics may be a promising solution. Microfluidic modified printing nozzles could facilitate the deposition of bioinks with a precisely tailored spatiotemporal composition in a mild fabrication environment, which could enhance the accuracy and printing quality. Utilizing microfluidic channels and chambers as the receiving plate could enable $3 \mathrm{D}$ printing on a chip and optimize the course of the printing and culture of, administration of stimuli to, and detection of the responses of $3 \mathrm{D}$ constructs. The introduction of the microchannel concept into built-in intracomplex architectures helps the generation of tissues/organs containing a microscale flow network, in which the form and function are more closely related to those of in vivo tubular structures, especially blood vessels. 


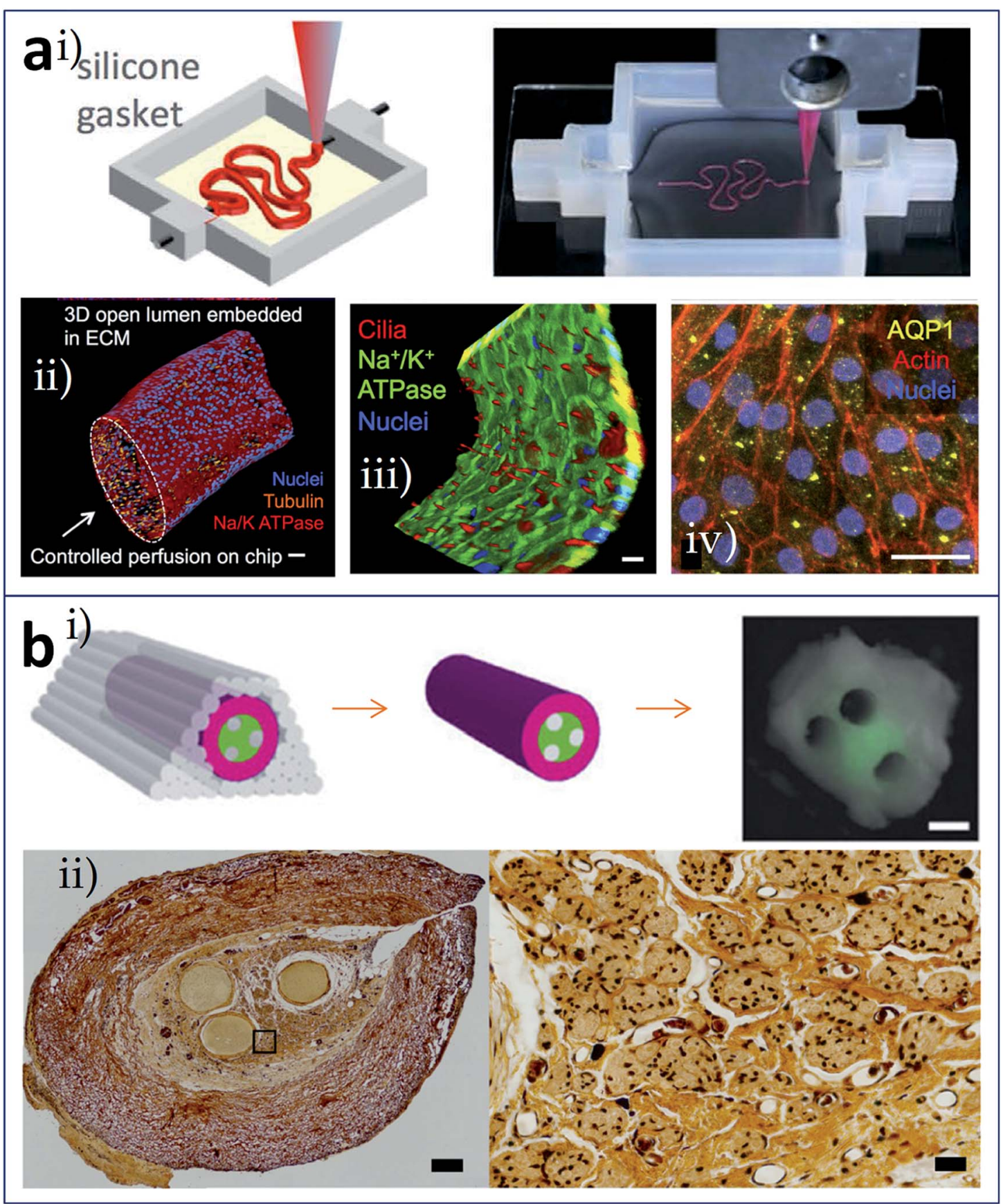

Fig. 6 Bioprinting of tissues/organs with built-in microchannels mimicking (a) renal tubules and (b) nerve conduits. (a-i) Schematic and photograph of the printing step during the preparation of 3D convoluted and perfusable proximal tubules, in which Pluronic F127 was printed on a gelatin-fibrinogen layer; (a-ii) 3D view of the renal proximal tubule with an open lumen structure, which was circumscribed with an epithelial lining and could be directionally perfused on the chip; red: Na/K ATPase, orange: acetylated tubulin, and blue: nuclei, scale bar is 50 um; (a-iii) partial tubule showing the apical side, highlighting the primary cilia (red), scale bar is $20 \mu \mathrm{m}$; (a-iv) proximal tubule showing actin (red) and AQP1 (yellow), scale bar is $20 \mu \mathrm{m}$. (b-i) Schematic and photograph of the resulting nerve graft, red: bioink composed of BMSC, green: bioink comprised of $90 \%$ BMSC and $10 \%$ SCs, grey: agarose rods; (b-ii) histological sections of the fabricated grafts (left panel, scale bar is $200 \mu \mathrm{m}$ ) and the axons shown as black dots (right panel, scale bar is $40 \mu \mathrm{m}$ ). Reproduced with permission from ref. 62 and 63 .

Compared to related classical and contemporary microfluidic technologies, as indicated in Fig. 1e-g, i.e., the formation of 3D cell-laden hydrogel constructs with tunable geometries via microchannel constrictions, ${ }^{29}$ bottom-up engineering of hydrogel building blocks, ${ }^{30}$ and the assembly of 3D constructs from microfluidic spun microfibres (e.g., via reeling, weaving, or direct writing), ${ }^{31-33}$ microfluidics-assisted bioprinting for construction of 3D tissues/organs shows unique advantages: (i) on the premise of elaborate structure construction, the volume accumulation and proportional enlargement of tissue blocks can be realized, which may be closer to the real tissues and organs in size; (ii) due to the combination of fine processing 
capacity in 3D bioprinting, the complexity of the microfluidics design, preparation and operation in microfluidics-assisted bioprinting would significantly reduce; (iii) compared with the relatively closed and limited space in most microfluidic systems, the samples from microfluidics-assisted bioprinting are easier to be recovered and used for subsequent protein and gene analysis, thus contributing to the mechanism study; (iv) the combination facilitates integration and matching of microfluidic control with peripheral equipment, especially automatic analysis equipment.

Recently, the most widely anticipated direction for development comprises the formation of an integrated organ-on-a-chip via one-piece bioprinting. Since the research carried out by Donald et al. on a lung-on-a-chip was published in Science in $2010,{ }^{64}$ organ-on-a-chip technology has attracted increasing attention as a new technology. Various organ-on-a-chip models have since been reported to simulate the internal environments of real organs, including blood vessel, liver, heart, and tumor chips. The complete range of elements involved in an organ-ona-chip include a microfluidic chip, cells or microtissues cultured in the chip, stimulus-applying components, and sensors for the readout of results. ${ }^{65}$ With the benefit of $3 \mathrm{D}$ printing, researchers have successfully prepared novel organson-chips that integrated all these elements (at least, a microfluidic device and the living constructs present in it) in a single continuous procedure using one printer. In the research carried out by Lee et al. (Fig. 7a), heterotypic cells and biomaterials were positioned for the formation of an organ-on-a-chip with no need for a secondary cell seeding process. ${ }^{66}$ The $3 \mathrm{D}$ bioprinting for liver-on-a-chip included steps as printing of poly ( $\varepsilon$-caprolactone) (PCL) cavity, printing of cells-ECM mixture, printing of cells-ECM mixture or enclosing PCL channel walls, and printing of tube connection part for perfusion. The schematic of the 3D bioprinted liver-on-a-chip is shown in Fig. 7a(i) and (ii) demonstrates a 3D/3D vertical co-culture model with HepG2 and HUVECs. More remarkably, Lind et al. fabricated cardiac devices via multi-material 3D printing (Fig. 7b). ${ }^{67}$ They sequentially printed six functional materials as components of a comprehensive cardiac chip to test contractile stresses and study the response of cardiac tissues to drugs. The operating principle of the cardiac devices is that anisotropic cardiac tissue contraction induces cantilever deflection, which is received by

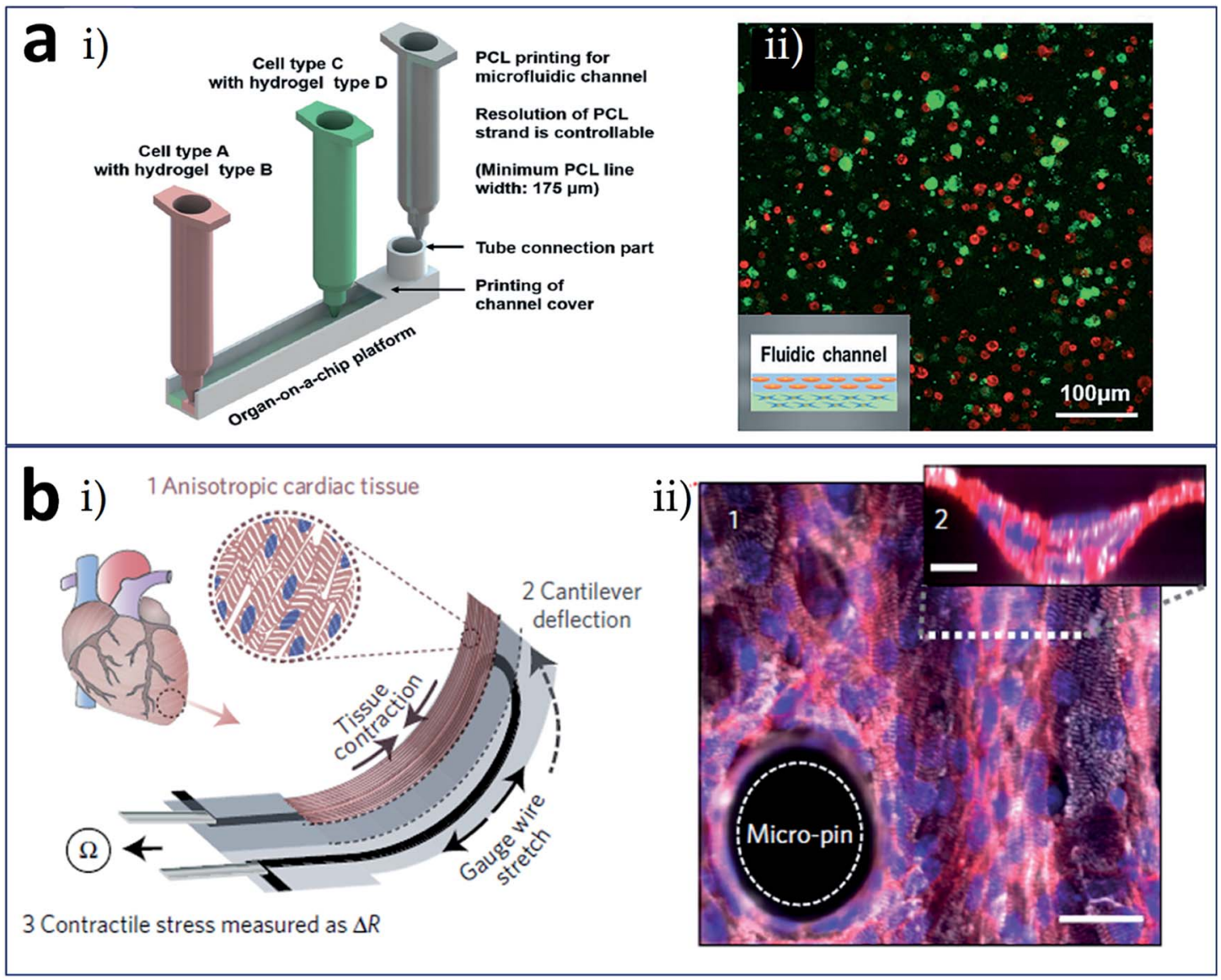

Fig. 7 Integrated (a) liver-on-a-chip and (b) heart-on-a-chip formed via one-piece bioprinting. (a-i) Schematic of the 3D bioprinting for liveron-a-chip, including steps as printing of PCL cavity, printing of cells-ECM mixture, printing of cells-ECM mixture or enclosing PCL channel walls, and printing of tube connection part for perfusion; (a-ii) a 3D/3D vertical model characterized by co-culturing of HepG2 and HUVECs. (b-i) Schematic of the device principle: anisotropic cardiac tissue contraction (1) induces cantilever deflection (2), which is received by the gauge wire in the cantilever; the stretching of gauge wire produces a resistance change used for the measure of tissue contractile stress (3); (b-ii) immunostained laminar tissue on the cantilever surface modified with micro-pin and micro-well structures, white: $\boldsymbol{\alpha}$-actinin, red: actin, and blue: nuclei, scale bar in the bottom is $30 \mu \mathrm{m}$. Reproduced with permission from ref. 66 and 67. 
Table 1 Examples of bioprinted 3D tissues/organs combined with microfluidics

Bioprinting-microfluidics combination manners

Extrusion-based bioprinting with microfluidic modified printing nozzle

Extrusion-based bioprinting with microfluidic modified printing nozzle

Extrusion-based bioprinting with microfluidic modified printing nozzle

Extrusion-based bioprinting with microfluidic modified printing nozzle Extrusion-based bioprinting with microfluidic modified printing nozzle Extrusion-based bioprinting with microfluidic modified printing nozzle

Extrusion-based bioprinting with microfluidic modified printing nozzle

Extrusion-based bioprinting with cell printing in the receiving microfluidic plate Inkjet bioprinting with cell printing in the receiving microfluidic plate

Inkjet bioprinting with cell printing in the receiving microfluidic plate

Extrusion-based bioprinting with cell printing in the receiving microfluidic plate Inkjet bioprinting with cell printing in the receiving microfluidic plate Sacrificial layer process and extrusion based bioprinting of constructs with built-in microchannels

Sacrificial layer process and extrusion based bioprinting of constructs with built-in microchannels

Sacrificial layer process and inkjet based bioprinting of constructs with built-in microchannels

Sacrificial layer process and inkjet based bioprinting of constructs with built-in microchannels Sacrificial layer process and extrusion based bioprinting of constructs with built-in microchannels

Sacrificial layer process and extrusion based bioprinting

\begin{tabular}{|c|c|c|c|}
\hline Cell types & Matrix materials & Target tissues/organs & Ref. \\
\hline Cartilage progenitor cells & Alginate & Vascular tissues & 35 \\
\hline $\begin{array}{l}\text { Human coronary artery } \\
\text { smooth muscle cells }\end{array}$ & $\begin{array}{l}\text { Carbon-nanotube reinforced } \\
\text { alginate }\end{array}$ & Vascular conduits & 36 \\
\hline L929 mouse fibroblasts & Alginate & $\begin{array}{l}\text { Lager-scale organs with } \\
\text { built-in microchannels }\end{array}$ & 37 \\
\hline $\begin{array}{l}\text { L929 mouse fibroblasts, } \\
\text { smooth muscle cells, } \\
\text { endothelial cells }\end{array}$ & Alginate & $\begin{array}{l}\text { Vascular circulation flow } \\
\text { system }\end{array}$ & 38 \\
\hline $\begin{array}{l}\text { Human umbilical vein } \\
\text { endothelial cells (HUVECs) }\end{array}$ & Alginate & Vascular network & 39 \\
\hline $\begin{array}{l}\text { Human embryonic kidney } \\
\text { cells }\end{array}$ & Alginate & Soft tissue scaffolds & 40 \\
\hline
\end{tabular}

(C2C12)

Hepatocellular carcinoma

Alginate

Liver

(HepG2) cells

Hepatocytes and endothelial

Fibronectin-gelatin

Liver

HepG2/C3A cells

Gelatin methacryloyl

Liver

HepG2 cells

Alginate

Liver

Hepatoma and glioma cells

Alginate

Liver

48

Not mentioned in the original work

Pluronic F127-diacrylate, (sacrificial material: Pluronic F127)

Endothelial cells, 10T1/2 cells, primary hepatocytes, stromal fibroblasts

Agarose, alginate, PEG, fibrin, matrigel, (sacrificial material: carbohydrate glass)

\section{HUVECs}

Collagen, (sacrificial material: gelatin)

HUVECs, normal human lung fibroblasts

Collagen, fibrin, (sacrificial material: gelatin)

HepG2, NIH3T3, mouse calvarial pre-osteoblasts (MC3T3) cells

HepG2/C3A cells, HUVECs
GelMA, star poly (ethylene glycol-co-lactide) acrylate (SPELA), poly (ethylene glycol) dimethacrylate (PEGDMA), poly (ethylene glycol) diacrylate (PEGDA), (sacrificial material: agarose) GelMA, (sacrificial material: agarose)
Microvascular networks

Vascular tissues

Vascular tissues

Vascular tissues

Vascular tissues 
Table 1 (Contd.)

Bioprinting-microfluidics combination manners Cell types Matrix materials Target tissues/organs Ref.

of constructs with built-in microchannels

Sacrificial layer process and extrusion based bioprinting of constructs with built-in microchannels Sacrificial layer process and extrusion based bioprinting of constructs with built-in microchannels Stereolithographic bioprinting of constructs with built-in microarchitecture Stereolithographic bioprinting of constructs with built-in microarchitecture

Block assembly and extrusion based bioprinting of constructs with built-in microchannels

Sacrificial layer process and extrusion based bioprinting of constructs with built-in microchannels

Block assembly and extrusion based bioprinting of constructs with built-in microchannels

One-step fabrication of an organ-on-a-chip using cell/ biomaterial printing One-step fabrication of an organ-on-a-chip using cell/ biomaterial printing
Human mesenchymal stem cells (hMSCs),human neonatal dermal fibroblasts (hNDFs), HUVECs C3H/10T1/2, hNDFs, HUVECS

HUVECs, C3H/10T1/2 cells, HepG2 cells

Human induced pluripotent stem cells (hiPSCs) derived hepatic cells, HUVECs, adipose-derived stem cells (ADSCs)

Chinese hamster ovary

(CHO) cells, human umbilical vein smooth muscle cells (HUVSMCs), human skin fibroblasts (HSFs), porcine aortic smooth muscle cells (PASMCs)

Human immortalized PTEC cells

Bone marrow stem cells (BMSC), Schwann cells (SCs)

HepG2 cells, HUVECs

Neonatal rat ventricular myocytes (NRVMs), human induced pluripotent stem cell-derived cardiomyocytes (hiPS-CMs)
Fibrin, gelatin, (sacrificial material: Pluronic F127)

GelMA, (sacrificial material: Pluronic F127)

Glycidal methacrylatehyaluronic acid (GM-HA), GelMA

GM-HA, GelMA

Agarose (also as the sacrificial material)

Gelatin, fibrin, (sacrificial material: Pluronic F127)

Agarose (also as the sacrificial material)

Poly( $\varepsilon$-caprolactone) (PCL), gelatin, collagen

Polydimethylsiloxane (PDMS)
Thick vascularized tissues

57

Vascularized tissues

Vascularized tissues

59

Vascularized hepatic constructs

60

Vascular tissues

61

Renal proximal tubules

62

Nerve conduit

63

Liver

66

Cardiac tissues

67 the gauge wire in the cantilever; the stretching of gauge wire produces a resistance change used for the measure of tissue contractile stress (Fig. 7b(i)). To support thick laminar tissues, the cantilever surface could be modified with micro-pin and micro-well structures (Fig. 7b(ii)). Moreover, a bioprinting technique combined with a microfluidic control enable functionally graded (multi-biomaterial and/or multicellular) additive manufacturing, which represents a promising strategy for future 4D bioprinting. Together with developments and breakthroughs in research into microfabrication technology, biomaterials, stem cells, and physiological microenvironments, it is expected that 3D bioprinting technology can be used to construct tissues and organs with functional activities and apply them in the fields of tissue engineering, regenerative therapy, organ transplantation, and high-throughput drug screening. Interestingly, inspired by the idea of tissues/organs printing, the combination of 3D printing and microfluidic technologies is expected to print artificial food with precisely controlled components (cells, biological factors etc.), which could be used as food safety and quality detection models. ${ }^{68}$

Table 1 summarizes the bioprinting-microfluidics combination manners, cell types, matrix materials, and target tissues/ organs of the bioprinted 3D tissues/organs that are discussed in this review.

\section{Conflicts of interest}

There are no conflicts to declare. 


\section{Acknowledgements}

This work was funded by the National Natural Science Foundation of China (No. 81271412, 81471308, and 31500793), and the Doctoral Scientific Research Foundation of Liaoning Province (No. 201501158).

\section{References}

1 A. Hart, J. M. Smith, M. A. Skeans, S. K. Gustafson, D. E. Stewart, W. S. Cherikh, J. L. Wainright, G. Boyle, J. J. Snyder, B. L. Kasiske and A. K. Israni, OPTN/SRTR Annual Data Report 2014: Kidney, Am. J. Transplant., 2016, 16(Suppl 2), 11-46.

2 R. Cortesini, Stem cells, tissue engineering and organogenesis in transplantation, Transplant Immunol., 2005, 15(2), 81-89.

3 S. Noh, N. Myung, M. Park, S. Kim, S.-U. Zhang and H.-W. Kang, 3D Bioprinting for Tissue Engineering, Clinical Regenerative Medicine in Urology, 2018, pp. 105-123.

4 Y. K. Kurokawa and S. C. George, Tissue engineering the cardiac microenvironment: Multicellular microphysiological systems for drug screening, Adv. Drug Delivery Rev., 2016, 96, 225-233.

5 L. Ferroni, A. Della Puppa, D. D'Avella, M. Isola, R. Scienza, C. Gardin and B. Zavan, Tissue Engineering Strategies as Tools for Personalized Meningioma Treatment, Artif. Organs, 2015, 39(7), E114-E126.

6 S. V. Murphy and A. Atala, 3D bioprinting of tissues and organs, Nat. Biotechnol., 2014, 32(8), 773-785.

7 C. Mandrycky, Z. Wang, K. Kim and D.-H. Kim, 3D bioprinting for engineering complex tissues, Biotechnol. Adv., 2016, 34(4), 422-434.

8 V. Mironov, T. Boland, T. Trusk, G. Forgacs and R. R. Markwald, Organ printing: computer-aided jet-based 3D tissue engineering, Trends Biotechnol., 2003, 21(4), 157161.

9 H.-W. Kang, S. J. Lee, I. K. Ko, C. Kengla, J. J. Yoo and A. Atala, A 3D bioprinting system to produce human-scale tissue constructs with structural integrity, Nat. Biotechnol., 2016, 34(3), 312-319.

10 M. M. Stanton, J. Samitier and S. Sánchez, Bioprinting of 3D hydrogels, Lab Chip, 2015, 15(15), 3111-3115.

11 A. C. Daly, G. M. Cunniffe, B. N. Sathy, O. Jeon, E. Alsberg and D. J. Kelly, 3D Bioprinting of Developmentally Inspired Templates for Whole Bone Organ Engineering, $A d v$. Healthcare Mater., 2016, 5(18), 2353-2362.

12 K. Markstedt, A. Mantas, I. Tournier, H. Martinez Avila, D. Hagg and P. Gatenholm, 3D Bioprinting Human Chondrocytes with Nanocellulose-Alginate Bioink for Cartilage Tissue Engineering Applications, Biomacromolecules, 2015, 16(5), 1489-1496.

13 W. Jia, P. S. Gungor-Ozkerim, Y. S. Zhang, K. Yue, K. Zhu, W. Liu, Q. Pi, B. Byambaa, M. R. Dokmeci, S. R. Shin and A. Khademhosseini, Direct 3D bioprinting of perfusable vascular constructs using a blend bioink, Biomaterials, 2016, 106, 58-68.
14 A. Faulkner-Jones, C. Fyfe, D.-J. Cornelissen, J. Gardner, J. King, A. Courtney and W. Shu, Bioprinting of human pluripotent stem cells and their directed differentiation into hepatocyte-like cells for the generation of mini-livers in 3D, Biofabrication, 2015, 7(4), 044102.

15 D. Singh, D. Singh and S. Han, 3D Printing of Scaffold for Cells Delivery: Advances in Skin Tissue Engineering, Polymers, 2016, 8(1), 19.

16 D. Mark, S. Haeberle, G. Roth, F. von Stetten and R. Zengerle, Microfluidic lab-on-a-chip platforms: requirements, characteristics and applications, Chem. Soc. Rev., 2010, 39(3), 1153.

17 G. Y. Huang, L. H. Zhou, Q. C. Zhang, Y. M. Chen, W. Sun, F. $\mathrm{Xu}$ and T. J. Lu, Microfluidic hydrogels for tissue engineering, Biofabrication, 2011, 3(1), 012001.

18 C. Colosi, S. R. Shin, V. Manoharan, S. Massa, M. Costantini, A. Barbetta, M. R. Dokmeci, M. Dentini and A. Khademhosseini, Microfluidic Bioprinting of Heterogeneous 3D Tissue Constructs Using Low-Viscosity Bioink, Adv. Mater., 2016, 28(4), 677-684.

19 F. Zheng, F. Fu, Y. Cheng, C. Wang, Y. Zhao and Z. Gu, Organ-on-a-Chip Systems: Microengineering to Biomimic Living Systems, Small, 2016, 12(17), 2253-2282.

20 J. B. Lee and J. H. Sung, Organ-on-a-chip technology and microfluidic whole-body models for pharmacokinetic drug toxicity screening, Biotechnol. J., 2013, 8(11), 1258-1266.

21 S. Waheed, J. M. Cabot, N. P. Macdonald, T. Lewis, R. M. Guijt, B. Paull and M. C. Breadmore, 3D printed microfluidic devices: enablers and barriers, Lab Chip, 2016, 16(11), 1993-2013.

22 A. A. Yazdi, A. Popma, W. Wong, T. Nguyen, Y. Pan and J. Xu, 3D printing: an emerging tool for novel microfluidics and lab-on-a-chip applications, Microfluid. Nanofluid., 2016, $20(3), 50$.

23 R. Amin, S. Knowlton, A. Hart, B. Yenilmez, F. Ghaderinezhad, S. Katebifar, M. Messina, A. Khademhosseini and S. Tasoglu, 3D-printed microfluidic devices, Biofabrication, 2016, 8(2), 022001.

24 K. Hölzl, S. Lin, L. Tytgat, S. Van Vlierberghe, L. Gu and A. Ovsianikov, Bioink properties before, during and after 3D bioprinting, Biofabrication, 2016, 8(3), 032002.

25 A. Arslan-Yildiz, R. E. Assal, P. Chen, S. Guven, F. Inci and U. Demirci, Towards artificial tissue models: past, present, and future of 3D bioprinting, Biofabrication, 2016, 8(1), 014103.

26 B. Derby, Printing and Prototyping of Tissues and Scaffolds, Science, 2012, 338(6109), 921-926.

27 A. V. Borovjagin, B. M. Ogle, J. L. Berry and J. Zhang, From Microscale Devices to 3D Printing, Circ. Res., 2017, 120(1), 150-165.

28 J. Ma, Y. Wang and J. Liu, Biomaterials Meet Microfluidics: From Synthesis Technologies to Biological Applications, Micromachines, 2017, 8(8), 255.

29 X. Zhang, Z. Meng, J. Ma, Y. Shi, H. Xu, S. Lykkemark and J. Qin, Flexible Fabrication of Shape-Controlled Collagen Building Blocks for Self-Assembly of 3D Microtissues, Small, 2015, 11(30), 3666-3675. 
$30 \mathrm{~J} . \mathrm{He}$, M. Mao, Y. Liu, L. Zhu and D. Li, Bottom-up fabrication of 3D cell-laden microfluidic constructs, Mater. Lett., 2013, 90, 93-96.

31 T. Sun, Q. Huang, Q. Shi, H. Wang, X. Liu, M. Seki, M. Nakajima and T. Fukuda, Magnetic assembly of microfluidic spun alginate microfibers for fabricating three-dimensional cell-laden hydrogel constructs, Microfluid. Nanofluid., 2015, 19(5), 1169-1180.

32 H. Onoe, T. Okitsu, A. Itou, M. Kato-Negishi, R. Gojo, D. Kiriya, K. Sato, S. Miura, S. Iwanaga, K. KuribayashiShigetomi, Y. T. Matsunaga, Y. Shimoyama and S. Takeuchi, Metre-long cell-laden microfibres exhibit tissue morphologies and functions, Nat. Mater., 2013, 12(6), 584-590.

33 D. Wei, J. Sun, J. Bolderson, M. Zhong, M. J. Dalby, M. Cusack, H. Yin, H. Fan and X. Zhang, Continuous Fabrication and Assembly of Spatial Cell-Laden Fibers for a Tissue-Like Construct via a Photolithographic-Based Microfluidic Chip, ACS Appl. Mater. Interfaces, 2017, 9(17), 14606-14617.

34 J. Rouwkema, N. C. Rivron and C. A. van Blitterswijk, Vascularization in tissue engineering, Trends Biotechnol., 2008, 26(8), 434-441.

35 Y. Zhang, Y. Yu, H. Chen and I. T. Ozbolat, Characterization of printable cellular micro-fluidic channels for tissue engineering, Biofabrication, 2013, 5(2), 025004.

36 F. Dolati, Y. Yu, Y. Zhang, A. M. D. Jesus, E. A. Sander and I. T. Ozbolat, In vitroevaluation of carbon-nanotubereinforced bioprintable vascular conduits, Nanotechnology, 2014, 25(14), 145101.

37 Q. Gao, Y. He, J.-z. Fu, A. Liu and L. Ma, Coaxial nozzleassisted 3D bioprinting with built-in microchannels for nutrients delivery, Biomaterials, 2015, 61, 203-215.

38 Q. Gao, Z. Liu, Z. Lin, J. Qiu, Y. Liu, A. Liu, Y. Wang, M. Xiang, B. Chen, J. Fu and Y. He, 3D Bioprinting of Vessel-like Structures with Multilevel Fluidic Channels, ACS Biomater. Sci. Eng., 2017, 3(3), 399-408.

39 R. Attalla, C. Ling and P. Selvaganapathy, Fabrication and characterization of gels with integrated channels using 3D printing with microfluidic nozzle for tissue engineering applications, Biomed. Microdevices, 2016, 18(1), 17.

40 S. Ghorbanian, M. A. Qasaimeh, M. Akbari, A. Tamayol and D. Juncker, Microfluidic direct writer with integrated declogging mechanism for fabricating cell-laden hydrogel constructs, Biomed. Microdevices, 2014, 16(3), 387-395.

41 M. Costantini, S. Testa, P. Mozetic, A. Barbetta, C. Fuoco, E. Fornetti, F. Tamiro, S. Bernardini, J. Jaroszewicz, W. Święszkowski, M. Trombetta, L. Castagnoli, D. Seliktar, P. Garstecki, G. Cesareni, S. Cannata, A. Rainer and C. Gargioli, Microfluidic-enhanced 3D bioprinting of aligned myoblast-laden hydrogels leads to functionally organized myofibers in vitro and in vivo, Biomaterials, 2017, 131, 98-110.

42 H.-G. Yi, H. Lee and D.-W. Cho, 3D Printing of Organs-OnChips, Bioengineering, 2017, 4(1), 10.
43 R. Chang, J. Nam and W. Sun, Direct Cell Writing of 3D Microorgan for In Vitro Pharmacokinetic Model, Tissue Eng., Part C, 2008, 14(2), 157-166.

44 R. Chang, K. Emami, H. Wu and W. Sun, Biofabrication of a three-dimensional liver micro-organ as anin vitrodrug metabolism model, Biofabrication, 2010, 2(4), 045004.

45 M. Matsusaki, K. Sakaue, K. Kadowaki and M. Akashi, ThreeDimensional Human Tissue Chips Fabricated by Rapid and Automatic Inkjet Cell Printing, Adv. Healthcare Mater., 2013, 2(4), 534-539.

46 N. S. Bhise, V. Manoharan, S. Massa, A. Tamayol, M. Ghaderi, M. Miscuglio, Q. Lang, Y. Shrike Zhang, S. R. Shin, G. Calzone, N. Annabi, T. D. Shupe, C. E. Bishop, A. Atala, M. R. Dokmeci and A. Khademhosseini, A liver-on-a-chip platform with bioprinted hepatic spheroids, Biofabrication, 2016, 8(1), 014101.

47 J. Snyder, A. Rin Son, Q. Hamid and W. Sun, Fabrication of Microfluidic Manifold by Precision Extrusion Deposition and Replica Molding for Cell-Laden Device, J. Manuf. Sci. Eng., 2015, 138(4), 041007.

48 J. Zhang, F. Chen, Z. He, Y. Ma, K. Uchiyama and J.-M. Lin, A novel approach for precisely controlled multiple cell patterning in microfluidic chips by inkjet printing and the detection of drug metabolism and diffusion, Analyst, 2016, 141(10), 2940-2947.

49 E. C. Novosel, C. Kleinhans and P. J. Kluger, Vascularization is the key challenge in tissue engineering, Adv. Drug Delivery Rev., 2011, 63(4-5), 300-311.

$50 \mathrm{~W}$. Wu, A. DeConinck and J. A. Lewis, Omnidirectional Printing of 3D Microvascular Networks, Adv. Mater., 2011, 23(24), H178-H183.

51 J. S. Miller, K. R. Stevens, M. T. Yang, B. M. Baker, D. H. Nguyen, D. M. Cohen, E. Toro, A. A. Chen, P. A. Galie, X. Yu, R. Chaturvedi, S. N. Bhatia and C. S. Chen, Rapid casting of patterned vascular networks for perfusable engineered three-dimensional tissues, Nat. Mater., 2012, 11(9), 768-774.

52 V. K. Lee, D. Y. Kim, H. Ngo, Y. Lee, L. Seo, S.-S. Yoo, P. A. Vincent and G. Dai, Creating perfused functional vascular channels using 3D bio-printing technology, Biomaterials, 2014, 35(28), 8092-8102.

53 V. K. Lee, A. M. Lanzi, H. Ngo, S.-S. Yoo, P. A. Vincent and G. Dai, Generation of Multi-scale Vascular Network System Within 3D Hydrogel Using 3D Bio-printing Technology, Cell. Mol. Bioeng., 2014, 7(3), 460-472.

54 L. E. Bertassoni, J. C. Cardoso, V. Manoharan, A. L. Cristino, N. S. Bhise, W. A. Araujo, P. Zorlutuna, N. E. Vrana, A. M. Ghaemmaghami, M. R. Dokmeci and A. Khademhosseini, Direct-write bioprinting of cell-laden methacrylated gelatin hydrogels, Biofabrication, 2014, 6(2), 024105.

55 L. E. Bertassoni, M. Cecconi, V. Manoharan, M. Nikkhah, J. Hjortnaes, A. L. Cristino, G. Barabaschi, D. Demarchi, M. R. Dokmeci, Y. Yang and A. Khademhosseini, Hydrogel bioprinted microchannel networks for vascularization of 
tissue engineering constructs, Lab Chip, 2014, 14(13), 22022211.

56 S. Massa, M. A. Sakr, J. Seo, P. Bandaru, A. Arneri, S. Bersini, E. Zare-Eelanjegh, E. Jalilian, B.-H. Cha, S. Antona, A. Enrico, Y. Gao, S. Hassan, J. P. Acevedo, M. R. Dokmeci, Y. S. Zhang, A. Khademhosseini and S. R. Shin, Bioprinted 3D vascularized tissue model for drug toxicity analysis, Biomicrofluidics, 2017, 11(4), 044109.

57 D. B. Kolesky, K. A. Homan, M. A. Skylar-Scott and J. A. Lewis, Three-dimensional bioprinting of thick vascularized tissues, Proc. Natl. Acad. Sci. U. S. A., 2016, 113(12), 3179-3184.

58 D. B. Kolesky, R. L. Truby, A. S. Gladman, T. A. Busbee, K. A. Homan and J. A. Lewis, 3D Bioprinting of Vascularized, Heterogeneous Cell-Laden Tissue Constructs, Adv. Mater., 2014, 26(19), 3124-3130.

59 W. Zhu, X. Qu, J. Zhu, X. Ma, S. Patel, J. Liu, P. Wang, C. S. E. Lai, M. Gou, Y. Xu, K. Zhang and S. Chen, Direct 3D bioprinting of prevascularized tissue constructs with complex microarchitecture, Biomaterials, 2017, 124, 106115.

60 X. Ma, X. Qu, W. Zhu, Y.-S. Li, S. Yuan, H. Zhang, J. Liu, P. Wang, C. S. E. Lai, F. Zanella, G.-S. Feng, F. Sheikh, S. Chien and S. Chen, Deterministically patterned biomimetic human iPSC-derived hepatic model via rapid 3D bioprinting, Proc. Natl. Acad. Sci. U. S. A., 2016, 113(8), 2206-2211.
61 C. Norotte, F. S. Marga, L. E. Niklason and G. Forgacs, Scaffold-free vascular tissue engineering using bioprinting, Biomaterials, 2009, 30(30), 5910-5917.

62 K. A. Homan, D. B. Kolesky, M. A. Skylar-Scott, J. Herrmann, H. Obuobi, A. Moisan and J. A. Lewis, Bioprinting of 3D Convoluted Renal Proximal Tubules on Perfusable Chips, Sci. Rep., 2016, 6(1), 34845.

63 C. M. Owens, F. Marga, G. Forgacs and C. M. Heesch, Biofabrication and testing of a fully cellular nerve graft, Biofabrication, 2013, 5(4), 045007.

64 D. Huh, B. D. Matthews, A. Mammoto, M. Montoya-Zavala, H. Y. Hsin and D. E. Ingber, Reconstituting Organ-Level Lung Functions on a Chip, Science, 2010, 328(5986), 16621668.

65 Q. Yang, Q. Lian and F. Xu, Perspective: Fabrication of integrated organ-on-a-chip via bioprinting, Biomicrofluidics, 2017, 11(3), 031301.

66 H. Lee and D.-W. Cho, One-step fabrication of an organ-on-achip with spatial heterogeneity using a 3D bioprinting technology, Lab Chip, 2016, 16(14), 2618-2625.

67 J. U. Lind, T. A. Busbee, A. D. Valentine, F. S. Pasqualini, H. Yuan, M. Yadid, S. J. Park, A. Kotikian, A. P. Nesmith, P. H. Campbell, J. J. Vlassak, J. A. Lewis and K. K. Parker, Instrumented cardiac microphysiological devices via multimaterial three-dimensional printing, Nat. Mater., 2017, 16(3), 303-308.

68 A. Escarpa, Lights and shadows on Food Microfluidics, Lab Chip, 2014, 14(17), 3213-3224. 